\title{
Fever and abdominal pain in a returning traveller
}

\author{
Amit Kishore MRCP(UK) MRCP(London), Syed Mehdi MBBS MRCP(UK), Milan Sapundzieski MD, \\ Roger C Prudham FRCP, Jimmy K Limdi MBBS MRCP(London) FRCP(Edin)
}

\section{CASE PRESENTATION}

A 39-year-old man of Pakistani descent, residing in the United Kingdom, presented to the emergency department with a one-week history of fever, chills, rigors, malaise, nausea, vomiting and diarrhea. He had recently returned from Pakistan, where he spent two weeks. His medical history was unremarkable, and he was not taking any regular medications.

His physical examination was normal, except for a temperature of $38.5^{\circ} \mathrm{C}$ and slight tenderness in the right upper quadrant. He was hemodynamically stable.
The patient's blood counts and electrolyte levels were normal. His liver chemistry revealed an alanine aminotransferase level of $275 \mathrm{U} / \mathrm{L}$, an alkaline phosphatase level of $173 \mathrm{U} / \mathrm{L}$ and an albumin level of $25 \mathrm{~g} / \mathrm{L}$; his C-reactive protein level was $144 \mathrm{mg} / \mathrm{L}$. His blood smears were negative for malaria species, and serology for acute viral hepatitis was negative. His chest radiograph was normal. The patient's stool was sent for Clostridium difficile toxin testing, and his blood, stool and urine samples were sent for culture. An urgent abdominal ultrasound scan was performed on the same day, which revealed the source of his pain. What is the diagnosis? 


\section{DIAGNOSIS}

Blood cultures showed the presence of Salmonella typhi, susceptible to amoxicillin/clavulanate, azithromycin and ceftriaxone, but resistant to amoxicillin and chloramphenicol.

The ultrasound scan revealed a thick-walled edematous gall bladder with pericholecystic fluid, but no stones and no biliary duct dilation - all of which suggested an acute acalculous cholecystitis (AAC).

The patient was treated with intravenous ceftriaxone, and his diarrhea and abdominal pain eventually subsided. His blood counts and chemistry normalized and, on day 12 , his $\mathrm{C}$-reactive protein levels improved to $22 \mathrm{mg} / \mathrm{L}$ at discharge.

Follow-up at six months revealed he was well. The patient's blood tests were normal and stool cultures were negative, suggesting he was not a carrier. A follow-up ultrasound scan on this occasion was entirely normal.

\section{DISCUSSION}

AAC is an acute necro-inflammatory disease of the gall bladder with a multifactorial pathogenesis, accounting for approximately $10 \%$ of cases of acute cholecystitis and with a mortality rate ranging between $6 \%$ and $67 \%$ (1). In its most classical form, it occurs in diabetic individuals; in the critically ill; in individuals with acute diseases such as burns, major trauma, postsurgical conditions and systemic infections; and in AIDS patients (in which cytomegalovirus and cryptosporidium are commonly associated organisms) $(2,3)$.

It is believed that gall bladder stasis and ischemia result in a local inflammatory response with endothelial injury, further ischemia and stasis, which results in concentration of bile salts and an increase in gall bladder mucosal damage. Perforation can occur in severe cases (4).

Fasting, dehydration and narcotic analgesia are risk factors that predispose to biliary stasis (5). AAC as a complication of typhoid fever is extremely rare in adults, although reported widely in the pediatric literature. To the best of our knowledge, ours is one of a few documented culture-proven cases of typhoid fever associated with AAC (5-9).

Ultrasonography is the investigation of choice, with a sensitivity of between $67 \%$ and $92 \%$ (2). Classical features of AAC are absence of gallstones or sludge, thickening of the gall bladder (greater than $3 \mathrm{~mm}$ ) with pericholecystic fluid and a positive Murphy's sign induced by the ultrasound probe. Abdominal computed tomography is sometimes a good adjunct to ultrasound, but radioisotope hepatobiliary imino-diacetic acid scan is the gold standard if ultrasound results are equivocal. During hepatobiliary imino-diacetic acid scanning, failure to opacify the gall bladder is the most sensitive and specific finding of cholecystitis (10).

The management of typhoidal AAC in adults is controversial, perhaps reflective of the relative dearth of medical literature. Review of the literature suggests that conservative management of otherwise uncomplicated typhoidal AAC in adults with intravenous fluid, antibiotics and close clinical observation is appropriate (5-9). Our patient responded well to these measures and did not require cholecystectomy.

AAC complicating typhoid fever appears to be rare, but has been reported in children and adolescents. This infrequently reported syndrome in adults probably reflects a lack of awareness of the condition and consequent under-reporting. In patients with AAC, a history of travel to a typhoid-endemic area is important for the early recognition and appropriate treatment of this condition to prevent subsequent complications. An early diagnosis and prompt therapy with antibiotics may be sufficient to treat uncomplicated typhoidal AAC without surgery.

\section{REFERENCES}

1. Savoca PE, Longo WE, Zucker KA, McMillen MM, Modlin IM. The increasing prevalence of acalculous cholecystitis in outpatients. Results of a 7-year study. Ann Surg 1990;211:433-7.

2. Kalliafas S, Ziegler DW, Flancbaum L, Choban PS. Acute acalculous cholecystitis: Incidence, risk factors, diagnosis, and outcome. Am Surg 1998;64:471-5.

3. Inian G, Kanagalakshmi V, Kuruvilla PJ. Acute acalculous cholecystitis: A rare complication of typhoid fever. Singapore Med J 2006;47:327-8

4. Janowitz P, Kratzer W, Zemmler T, Tudyka J, Wechsler JG. Gallbladder sludge: Spontaneous course and incidence of complications in patients without stones. Hepatology 1994;20:291-4

5. khan FY, Elouzi EB, Asif M. Acute acalculous cholecystitis complicating typhoid fever in adult patient: A case report and review of the literature. Travel Med Infect Dis 2009; 7:203-6.

6. Lothrop HA. Acute cholecystitis complicating typhoid fever. Ann Surg 1915;62:152-7.

7. Garg P, Singh R, Sharda A, Dadoo RC. Perforation in acute acalculus Salmonella cholecystitis. Trop Doct 1995;25:84.

8. Avalos ME, Cerulli MA, Lee RS. Acalculous acute cholecystitis due to Salmonella typhi. Dig Dis Sci 1992;37:1772-5.

9. Lai CH, Huang CK, Chin C, Lin HH, Chi CY, Chen HP. Acute acalculous cholecystitis: A rare presentation of typhoid fever in adults. Scand J Infect Dis 2006;38:196-200.

10. Westlake PJ, Hershfield NB, Kelly JK, et al. Chronic right upper quadrant pain without gallstones: Does HIDA scan predict outcome after cholecystectomy? Am J Gastroenterol 1990;85:986-90. 


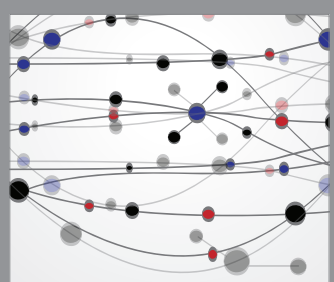

The Scientific World Journal
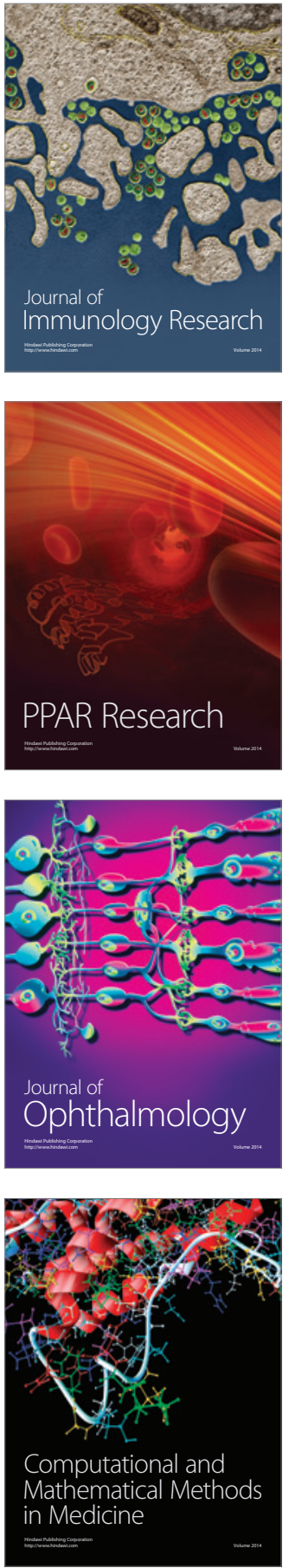

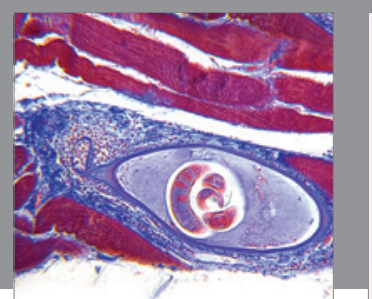

Gastroenterology Research and Practice

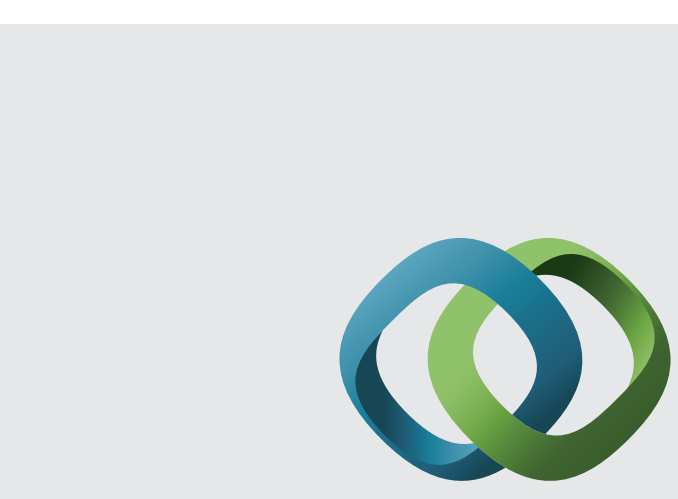

\section{Hindawi}

Submit your manuscripts at

http://www.hindawi.com
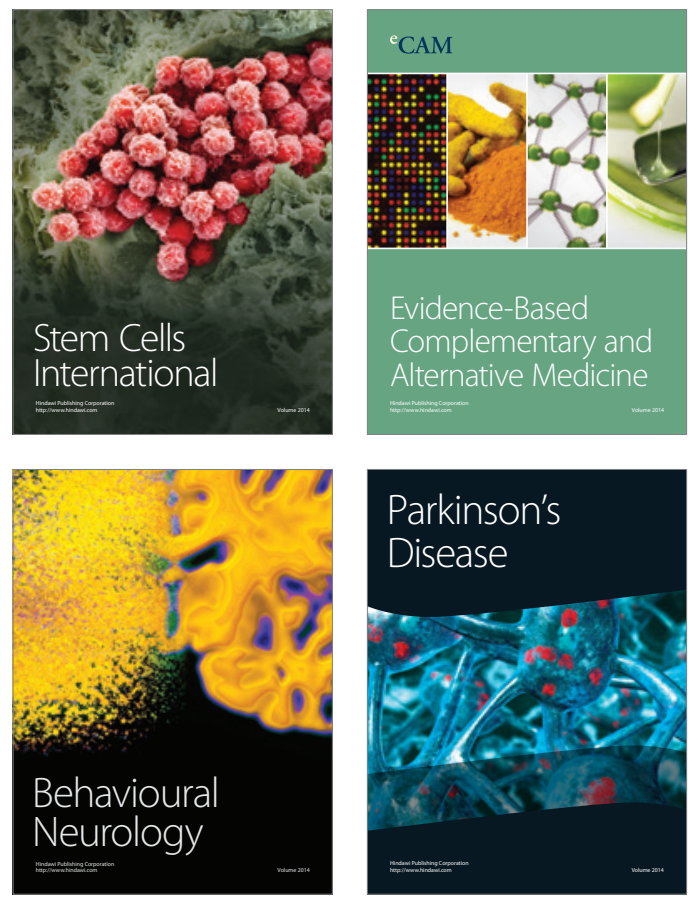
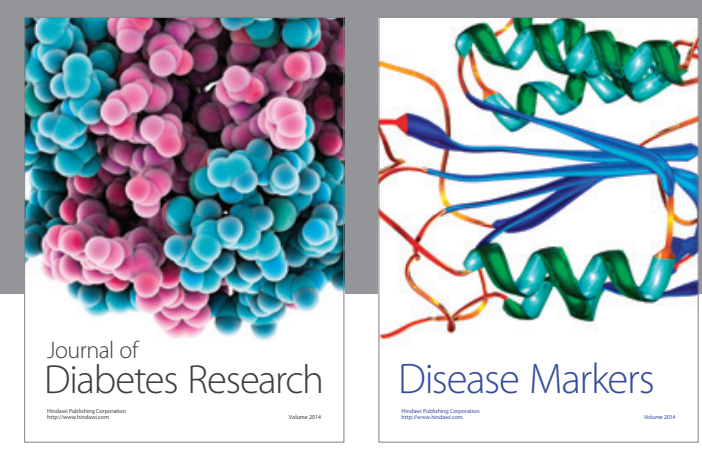

Disease Markers
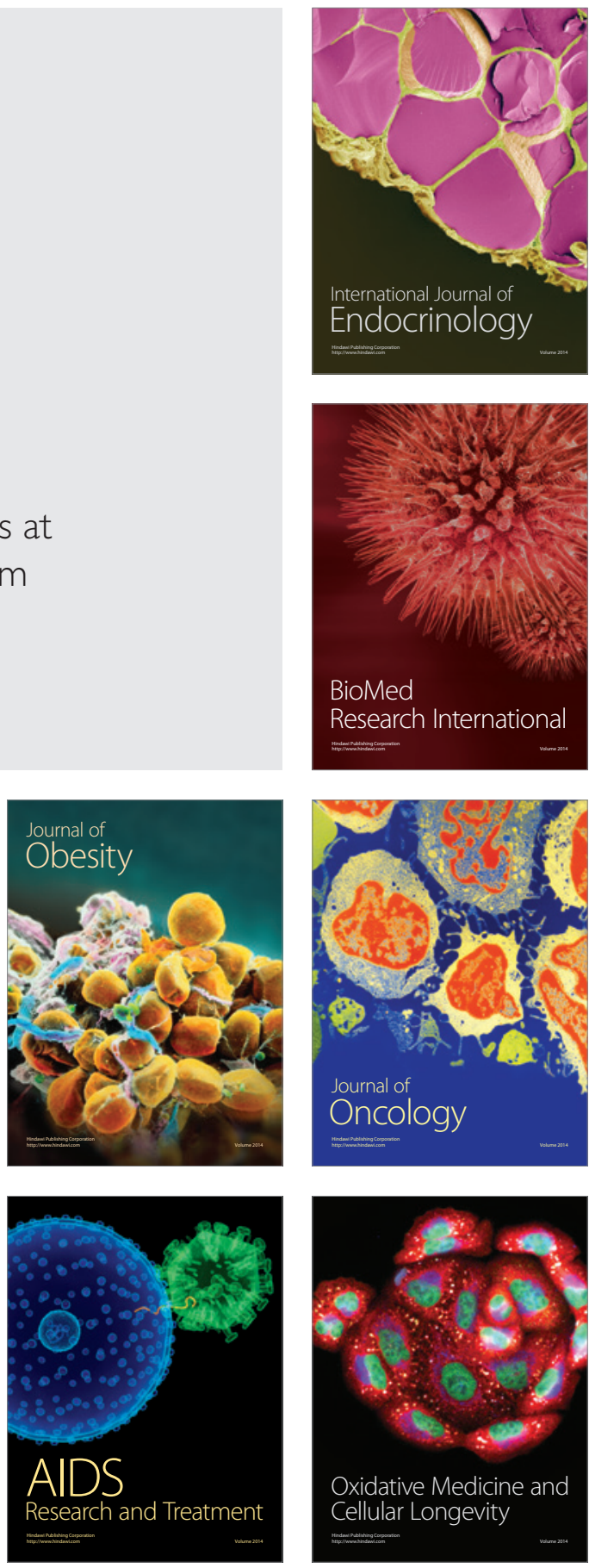